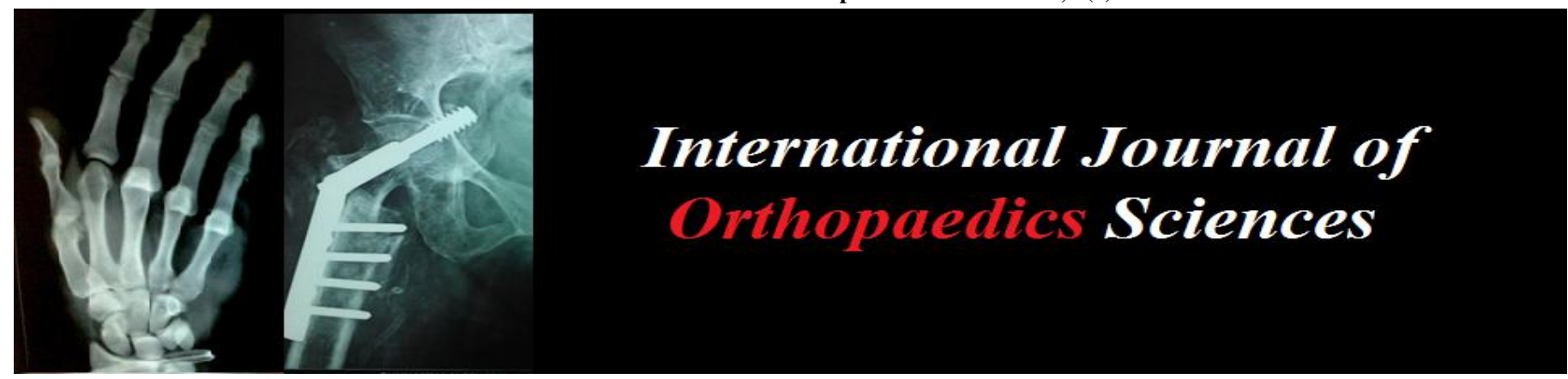

ISSN: $2395-1958$

IJOS 2017; 3(2): 181-185

(C) 2017 IJOS

www.orthopaper.com

Received: 28-02-2017

Accepted: 29-03-2017

Dr. Prakash Savakkanavar

Associate Prof of Orthopaedics, Sapthagiri Medical College,

Bangalore, India

Dr. Shekar M

Assistant professor of

Orthopaedics, Sanjay Gandhi

Institute of Trauma and

Orthopaedics, Bangalore, India

\section{Functional outcome of bilateral total knee arthroplasty: A two-year prospective study}

\author{
Dr. Prakash Savakkanavar and Dr. Shekar M
}

DOI: http://dx.doi.org/10.22271/ortho.2017.v3.i2c.28

\section{Abstract}

The wider adoption of simultaneous bilateral TKA has been limited due to the reports of the associated risk of mortality and perioperative complications. The present study evaluated the safety of single-stage bilateral total knee arthroplasty, in terms of perioperative morbidity, mortality and clinical outcomes of the procedure. The two-year prospective study was conducted on 100 knees of fifty patients who visited the Department of orthopaedics of a super speciality hospital, based in India. Detailed history was collected and clinical examination of the knees was performed in all the patients. Pre-and postoperative knee scores were calculated using modified Install's knee society scoring system. Postoperative knee society score was recorded up to 12 months of follow-up. Perioperative complications were noted in 8 subjects. Groups with and without complications were compared for various assessed variables, and the correlation between hospital stay and different demographic and clinical variables were studied by Spearman correlation. Out of the 50 subjects who underwent bilateral TKA, perioperative complications were noted in $16 \%$ of the subjects. Major complications like DVT, pulmonary embolism, myocardial infarction and death were not reported in any of the cases. A significant number of patients $(37.5 \%)$ belonging to the complication group underwent secondary procedures. The duration of hospital stay was found to be significantly associated with comorbidities, and perioperative complications. Simultaneous bilateral TKA is a safe procedure, providing satisfactory functional outcomes. Comorbidities of the patients has been noted as the major factor contributing to increased hospital stay

Keywords: Simultaneous bilateral TKA, staged bilateral TKA, functional outcomes, complications, comorbidities

\section{Introduction}

Total knee arthroplasty has emerged as a safe, cost-effective, and low-risk therapy for alleviating pain and achieving rehabilitation in arthritis patients [1]. Recent years have witnessed a significant increase in the annual number of total knee arthroplasties performed both in younger and elderly patients ${ }^{[1]}$. As per the 2010 estimates, the prevalence of subjects living with hip and knee arthroplasties in American population is around 7 million ${ }^{[2]}$.

For a patient requiring bilateral total knee arthroplasty, simultaneous and staged surgical procedures are available. Despite the advantages like less exposure to anaesthesia, costeffectiveness, and early return to full function, certain disadvantages limit the adoption of simultaneous bilateral TKA compared to unilateral TKA in clinical practice. These include associated risks such as higher rate of blood loss and pulmonary and fat embolisms, neurological, cardiac, and respiratory complications, and perioperative death ${ }^{[3,4]}$. However, some studies have reported similar rates of mortality, but increased morbidity associated with one-stage bilateral TKA, compared to unilateral TKA ${ }^{[5]}$. Certain studies have observed lesser rates of in-patient deaths among patients undergoing second arthroplasty in staged procedure, compared to one-stage procedure, with no difference in the rate of revision ${ }^{[6,7]}$.

The 2016 Canadian Institute for health information, comparing staged and simultaneous bilateral TKA, has reported that patients undergoing simultaneous TKA and functional recovery in both the knees had lesser risk of knee infection, shorter acute care stays, with no difference in rate of revision surgery ${ }^{[8]}$. In a recent study, Bagsby et al. showed no significant difference in the knee society score (KSS) among patients undergoing simultaneous and staged bilateral TKA. In addition, simultaneous bilateral TKA has been reported to offer significant post-operative functional benefits like increased knee flexion, total range of motion,
Correspondence

Dr. Prakash Savakkanavar Associate Prof of Orthopaedics, Sapthagiri Medical College,

Bangalore, India 
and function score in patients undergoing ${ }^{[9]}$. However, studies have demonstrated that elderly and high-risk patients undergoing simultaneous bilateral TKA, are at risk of developing systemic complications ${ }^{[10]}$. Some Indian-based studies recommend to reserve single-stage bilateral TKA, only in patients with advanced bilateral knee arthritis ${ }^{[3]}$. These literature findings indicate that there is no consensus on the outcome of single-stage bilateral TKA. The present study was intended to assess the safety of single-stage bilateral TKA in terms of perioperative morbidity and mortality and the clinical outcome of the procedure among Indian patients, in the light of limited literature evidence available from the country. In addition, the study attempted to identify the criteria for choosing patients for the bilateral single stage TKA.

\section{Materials and methods}

The prospective study was conducted at the Department of orthopaedics, in a super speciality hospital, based in India, between 2013 and 2015. Well-motivated subjects with American Society of Anaesthesiologists (APA) Grade I and II bilateral advanced arthritis, aged between 50- 80 yrs were recruited for the study. The study considered 100 knees of fifty patients. The exclusion criteria considered were subjects: aged over 80 years, belonging to ASA Grade III and IV, with uncontrolled diabetes, hypertensive with renal functional disturbances, neuropathy, mental illness, or with systemic and local infections. The study was approved by the institutional ethics committee. The nature, need, advantages, disadvantages, outcomes, and consequences of bilateral TKA were explained to all the participants and an informed consent was collected. Preanesthesia evaluation and fitness were assessed before the surgery.

A standard pro forma was used for collecting demographic and clinical characteristics such as age, sex, body mass index (BMI), brief history of diagnosis/comorbidities, length of hospital stay, number of blood transfusion, post-operative complications, infections, use of anticoagulants, secondary procedures, and length of ICU stay. KSS were recorded at 6 weeks, 3 months, 6 months, and 12 months post-operatively. Preoperative knee scores were calculated for both subjective and functional components, using modified Insall's knee society scoring system ${ }^{[11]}$. X- rays of standing anterioposterior and lateral views of both the knees were obtained. Routine blood tests, ECG, and 2D echo test were performed. In addition, special investigations like pulmonary function tests, and dobutamine stress echo test were done in relevant cases.

Subjects were operated under epidural and general anaesthesia. Anterior and posterior reference guides were set, and femoral and tibial components were implanted. Various implants (size ranging from 1.5-4 $\mathrm{mm}$ ) types used were: cruciate retaining $(\mathrm{CR})$ femoral prosthesis, cruciate sacrificing (CS) type, and cruciate sacrificing rotating platform (RPF). Average duration of the surgery was 3 hours 50 minutes. Repeated blood transfusions were performed in necessary subjects, depending on the postoperative hemoglobin levels. Postoperative epidural analgesia consisting of $0.1 \%$ sensorcaine and morphine was given continuously for 3 days. Pain was assessed using visual analogue scale (VAS). Postoperative pain protocol followed was oral analgesics for VAS score $>5$ and injectable analgesics for score $<5$. Deep vein thrombosis (DVT) prophylaxis was used only in subjects on regular aspirin, with history of varicose veins, and in those with difficulty in early mobilization. Chest physiotherapy and incentive spirometry were started one day prior to the surgery and continued during the postoperative period. The patients were made to stand and walk a few steps with walker support on first postoperative day. Quadriceps strengthening and knee bending exercises were also started immediately after surgery. Skin staples were removed between $10^{\text {th }}$ and $12^{\text {th }}$ postoperative days. The patients were advised to use walker support for 4 to 6 weeks. The patients were followed up at 6 weeks, 3 months, 6 months and 1 year. Secondary suturing was performed only in one patient who had wound healing problem.

Subjects were classified based on comorbidities and secondary procedures as present and absent groups. Based on the disease diagnosis, subjects were grouped as: patients with osteoarthritis (OA) and rheumatoid arthritis (RA). Comorbidities such as diabetes, hypertension, ischemic heart disease, peptic ulcer disease, hypothyroidism, asthma, and parkinsonism were noted among the subjects.

Continuous variables with normal distribution were represented as mean $\pm \mathrm{SD}$, and those without normal distribution as median (range). Categorical variables were represented as counts. Unpaired t-test was performed for continuous variables with normal distribution data with 2 groups and Mann-Whitney test for continuous variables without normal distribution data with 2 groups. Chi-square test was done for categorical variables. $\mathrm{P}$ value was assessed at $5 \%$ level of significance. Relationship between hospital stay and different variables were verified by Spearman correlation. MedCalc software was used for the analysis of the data.

\section{Results}

The study considered 100 knees of 50 enrolled subjects with an $\mathrm{M}$ : $\mathrm{F}$ ratio of $0.28: 1$. The mean age of the subjects was 61.28 years, ranging between $43-78$ years. Out of the 50 subjects, $42(84 \%)$ were suffering from OA and $8(16 \%)$ with RA. The mean BMI, pre-operative KSS, and preoperative $\mathrm{Hb} \%$ noted were: $27.92 \pm 3.85,29.56 \pm 12.56$ (3-63), and 12.4 g\% (10.2-15.4 g \%) respectively. Twenty-six patients belonged to ASA class I and 24 patients to ASA class II. The presence and absence of comorbidities were noted in 22 (44\%), and $28(56 \%)$ subjects respectively.

Secondary outcomes noted were: average postoperative $\mathrm{Hb} \%$ was $10.2 \mathrm{~g} \%$ (6.8g\%-12.6 g \%). Average blood transfusion was 1.64 units (1-8 units). The average duration of oxygen supplementation was 2.6 days (2-9 days). Three patients (6\%) required intensive care monitoring. The average duration of hospital stay was 11.6 days (5 -34 days).

Perioperative complications associated with bilateral TKA was noted in $8(16 \%)$ subjects, while $42(84 \%)$ subjects did not have complications. Minor postoperative complications including nausea and vomiting, ecchymosis, and urinary tract infection were noted in $8(16 \%), 14(28 \%)$, and $5(10 \%)$ subjects respectively. Other problems such as electrolyte imbalance and hematemesis, myalgia and weakness, confusion, dryness of mouth, pruritus, delayed wound healing, deep seated infection, and acute renal failure were noted in one case each. No death was reported. None of the patients showed symptoms suggestive of deep vein thrombosis, pulmonary embolism or myocardial infarction, and none of them were readmitted within 30 days.

The comparison of variables such as age, gender, BMI status, comorbidities, diagnosis, pre-operative knee scores, hospital stays, 3 months post-operative KSS, and secondary procedure between subjects with and without complications, showed a statistically significant relation between the group with respect to secondary procedures. A significant number of 
subjects undergoing secondary procedure belonged to the complication group, compared to subjects with no complications ( $\mathrm{P}$ 0.001). None of the patients without complications underwent secondary procedure. Even though there was a considerable variation in the mean number of days of hospital stay between the complications and no complications groups, the noted difference was not statistically significant, rather it showed a trend towards significance ( $P$ 0.0561). Patient with complications had an average hospital stay of 17.75 days compared to 10.5 days noted in patients without complications. The other evaluated variables did not show any significant difference among the groups (Table 1).

Table 1: Distribution of variables between groups with and without complications

\begin{tabular}{|c|c|c|c|}
\hline Variables & Complications $\mathrm{N}=8$ & No complications $\mathrm{N}=42$ & P value \\
\hline Age & $65.25 \pm 8.76$ & $60.52 \pm 8.56$ & 0.1603 \\
\hline Gender M/F & $2 / 6$ & $9 / 33$ & 0.8087 \\
\hline BMI & $27(24-34)$ & $28(22-36)$ & 0.9893 \\
\hline \multicolumn{4}{|c|}{ Comorbidities } \\
\hline yes & 5 & 17 & \multirow{2}{*}{0.4463} \\
\hline no & 3 & 25 & \\
\hline \multicolumn{4}{|c|}{ Diagnosis } \\
\hline $\mathrm{OA}$ & 7 & 35 & \multirow{2}{*}{0.8169} \\
\hline RA & 1 & 7 & \\
\hline Preoperative knee scores & $34.62 \pm 12.28$ & $28.59 \pm 12.04$ & 0.2017 \\
\hline Hospital stay & $17.75 \pm 8.82$ & $10.5 \pm 3.65$ & 0.0561 \\
\hline KSS postoperative 3 months & $82(75-90)$ & $86(60-90)$ & 0.3133 \\
\hline \multicolumn{4}{|c|}{ Secondary procedure } \\
\hline yes & 3 & 0 & \multirow{2}{*}{0.001} \\
\hline no & 5 & 42 & \\
\hline
\end{tabular}

A moderate correlation was noted between hospital stay and complications reported (r 0.361, P 0.01, CI 0.0921 to 0.581). A weak correlation was noted between hospital stay and variables like comorbidities ( $\mathrm{r} 0.282, \mathrm{P} 0.0471$, CI 0.00415 to 0.520 ), and secondary procedures ( $\mathrm{r} 0.164, \mathrm{P}$ 0.2542 , CI -0.119 to 0.423 ). The positive association of hospital stay with clinical variables like perioperative complications and comorbidities was noted to be statistically significant. A weak negative correlation was noted with variables like gender ( $\mathrm{r}-0.261)$, BMI ( $\mathrm{r}-0.133)$, and preoperative knee scores ( $\mathrm{r}-0.181)$. No correlation was noted with variables like age, diagnosis, and post-operative KSS (Table 2) (Fig. 1).

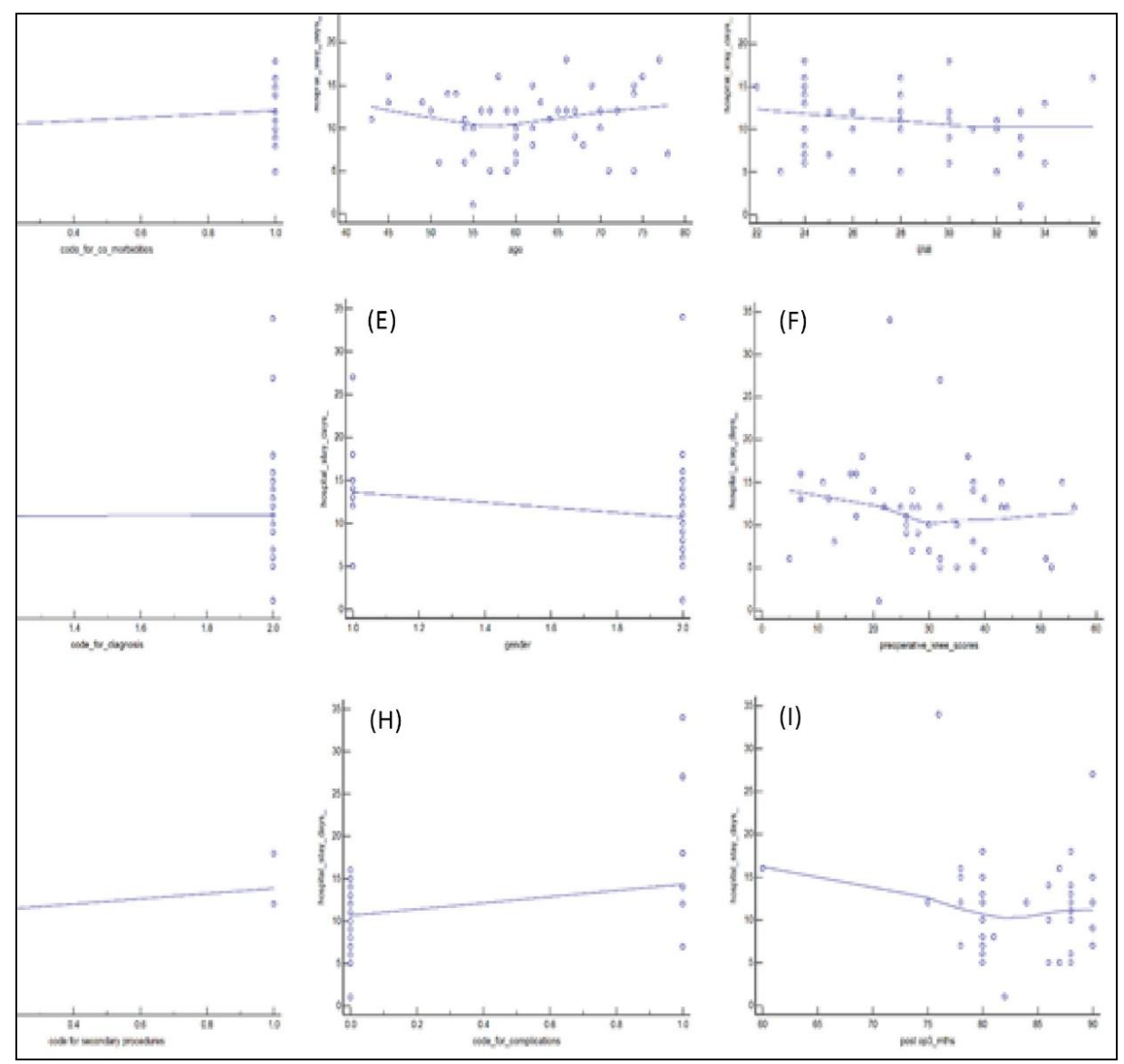

Fig 1: Graphs depicting correlation between hospital stay and various assessed variables (A) Comorbidities, (B) Age, (C) BMI, (D) Diagnosis (E) Gender, (F) Preoperative score, (G) Secondary procedure, (H) Complications, (I) Postop 3 months 
Table 2: Correlation between hospital stay and other variables

\begin{tabular}{|c|c|c|c|}
\hline Variables & Corre-coeff & 95\% confidence Interval & P value \\
\hline Age & 0.0911 & -0.192 to 0.360 & 0.5293 \\
\hline Gender M/F & -0.261 & -0.503 to 0.0190 & 0.0674 \\
\hline BMI & -0.133 & -0.397 to 0.151 & 0.3561 \\
\hline Comorbidities & 0.282 & 0.00415 to 0.520 & 0.0471 \\
\hline Diagnosis & 0.038 & -0.243 to 0.313 & 0.7932 \\
\hline Preoperative knee scores & -0.181 & -0.437 to 0.103 & 0.2088 \\
\hline Post-operative 3 months & -0.0656 & -0.338 to 0.217 & 0.6508 \\
\hline Complications & 0.361 & 0.0921 to 0.581 & 0.01 \\
\hline Secondary procedure & 0.164 & -0.119 to 0.423 & 0.2542 \\
\hline
\end{tabular}

Table 3: Evaluation of pre and postoperative KSS

\begin{tabular}{|c|c|c|c|c|c|}
\hline Evaluation & Number of patients & Range of motion & Mean \pm SD & $t^{*}$ & P* \\
\hline Preoperative & 50 & $5-56$ & $29.56 \pm 12.16$ & - & - \\
\hline \multicolumn{5}{|c|}{ Postoperative } \\
\hline 6 weeks & 50 & $55-88$ & $80.76 \pm 7.39$ & 27.214 & $<0.001$ \\
\hline 3 months & 50 & $60-90$ & $83.96 \pm 5.62$ & 29.801 & $<0.001$ \\
\hline 6 months & 43 & $75-90$ & $86.49 \pm 4.13$ & 29.774 & $<0.001$ \\
\hline 12 months & 13 & $80-90$ & $89.08 \pm 2.78$ & 20.904 & $<0.001$ \\
\hline
\end{tabular}

* Compared with preoperative score

A significant increase in range of motion from $5-56^{0}$ preoperative to $80-90^{\circ}, 12$ months postoperative was noted. The mean KSS had shown a significant increase from 29.56, preoperative to 80.76 six weeks postoperative $(P<0.001)$. Average postoperative knee score at $6^{\text {th }}$ month follow-up (86.4) was statistical significant in terms of functional outcome (Table 3 ). The comparison of pre and postoperative scores with age of the subjects, showed that the immediate postoperative knee scores were lower in elderly, but at $6^{\text {th }}$ month follow-up it was almost similar in all the age groups. The study also noted that males had better functional outcome than females.

\section{Discussion}

Literature review has substantiated the beneficial effects of one-stage bilateral TKA in reducing the duration of hospital stay and in enhancing the rehabilitation of the patients with severe bilateral knee arthritis. This is in concurrence with the present study finding ${ }^{[12]}$. The study has noted an increase in the mean KSS score from 29.56 to 80.76 within six weeks following arthroplasty. Average hospital stay was noted as11.66 days. Even though the hospital was ready to discharge the patients within 5 days after surgery, the slight increase in the duration of hospital stay noted in the study can be ascribed to the preference conveyed by some of the patients to leave the hospital after suture removal.

There are conflicting results on the risk of developing complications including pulmonary embolism, cardiac complications, and mortality among patients undergoing simultaneous bilateral TKA ${ }^{[13]}$. In the present study, none of the cases had mortality, or major complications like DVT, pulmonary embolism, or myocardial infraction. However, occurrence of minor complications was managed appropriately in eight patients.

The study by Lombardi et al, in 2001 reported that subjects aged $\geq 80$ years or more, undergoing simultaneous TKA are more prone to develop perioperative complications than subjects aged <80 years ${ }^{[14]}$. Similarly, Rahul et al in 2004 concluded that postoperative complications following TKA can be majorly suspected in patients belonging to ASA class III ${ }^{[15]}$. Whereas, the present study considered only up to 78 years' age groups, and included only subjects belonging to ASA grade I and grade II. Compared to subjects with no complications, the mean age was higher for those belonging to the complication group, even though the difference was not statistically significant (P 0.1603). The requirement for blood transfusion was found to be higher among patients undergoing simultaneous bilateral TKA, compared to the staged procedure ${ }^{[3]}$. Average blood transfusion was noted to be 1.64 units (1-8 units). This is comparable to the study by Sabari et $a l$, which reported the requirement of blood transfusion in $90 \%$ of the subjects, with about 1.7 units of blood transfused per patient.

Studies have reported increased incidence of neurological complications, predominately confusion among patients undergoing one-staged bilateral TKA ${ }^{[3]}$. Whereas in the current study, postoperative confusion had been reported only in one subject, which subsequently resolved with oxygen supplementation. In concurrence with this finding, an Indianbased study by Sabari et al. have reported the occurrence of confusion in 3 cases following simultaneous bilateral TKA, but no incidence of fat emboli or cardiovascular risk.

Acute renal failure has been reported a rare complication of postoperative, single-staged bilateral TKA. Similar to this literature finding, the present study has noted only one case of acute renal failure in a 70 -year-old patient ${ }^{[16]}$. The incidence of nausea and vomiting observed was $16 \%$, and could be mainly due to the use of morphine as the epidural infusion for postoperative analgesia. The condition was treated by withdrawing the drug and supplementing with other opioids. The incidence rate of urinary tract infection noted was $10 \%$. This could be attributed to the use of indwelling urinary catheter till the removal of epidural pump. These cases were treated with appropriate antibiotics. The present study suggests that the ideal Indian candidates for bilateral singlestage total knee replacement are those, aged $<70$ years, with bilateral symmetrical arthritis, belonging to ASA class I and II, and those who are well motivated.

\section{Conclusion}

Bilateral single stage procedure has definite advantages of reduced hospital stay and early rehabilitation of the patients suffering from severe bilateral knee arthritis. The mean postoperative knee scores were found to be satisfactory in all the study participants. To the best of our knowledge this is the first study from India that has evaluated the influence of 
comorbidities on the duration of hospital stay and the incidence of complications following simultaneous bilateral TKA. In addition, the study has shown that the risk of perioperative mortality, and cardiovascular, pulmonary, and neurological complications are comparatively lesser in patients undergoing bilateral TKA. However, it should be emphasized that for any surgical procedure, the ultimate result may depend in large part on the expertise of the surgeon and the established pattern of preoperative medical clearance and postoperative management.

\section{References}

1. NIH Consensus Panel. NIH Consensus Statement on total knee replacement December 8-10, 2003. J Bone Joint Surg Am. 2004; 86(6):1328-35.

2. Maradit Kremers H, Larson DR, Crowson CS, Kremers WK, Washington RE, Steiner CA et al. Prevalence of Total Hip and Knee Replacement in the United States. J Bone Joint Surg Am. 2015; 97(17):1386-97.

3. Girish SR, Gopalakrishnan KC, Sugath S, Anoop S. Simultaneous bilateral total knee arthroplasty - is it the final answer? Kerala Journal of Orthopaedics. 2011; 24(1):15-22.

4. Jankiewicz JJ, Sculco TP, Ranawat CS, Behr C, Tarrentino S. One-stage versus 2-stage bilateral total knee arthroplasty. Clin Orthop Relat Res. 1994; 309:94101.

5. Luscombe JC, Theivendran K, Abudu A, Carter SR. The relative safety of one-stage bilateral total knee arthroplasty. Int Orthop. 2009; 33(1):101-4.

6. Hussain N, Chien T, Hussain F, Bookwala A, Simunovic $\mathrm{N}$, Shetty V et al. Simultaneous Versus Staged Bilateral Total Knee Arthroplasty. HSS J. 2013; 9(1):50-9.

7. Bohm ER, Molodianovitsh K, Dragan A, Zhu N, Webster G, Masri B et al. Outcomes of unilateral and bilateral total knee arthroplasty in 238,373 patients. Acta Orthop. 2016; 87(1):24-30.

8. Canadian Institute for Health Information. Outcomes for Simultaneous and Staged Bilateral Total Knee Replacement Surgeries. Ottawa, ON: CIHI. 2016.

9. Bagsby D, Pierson JL. Functional outcomes of simultaneous bilateral versus unilateral total knee arthroplasty. Orthopaedics. 2015; 38(1):43-47.

10. Yoon HS, Han CD, Yang IH. Comparison of simultaneous bilateral and staged bilateral total knee arthroplasty in terms of perioperative complications. J Arthroplasty. 2010; 25(2):179-85.

11. Insall JN, Dorr LD, Scott RD, Scott WN. Rationale of the Knee Society clinical rating system. Clin Orthop Relat Res. 1989; 248:13-4.

12. MS CC, Patil S. Safety of bilateral single stage total knee replacement under one anaesthesia in terms of perioperative complications. International Journal of Current Research and Review. 2013; 5(7):102-10.

13. Restrepo C, Parvizi J, Dietrich T, Einhorn TA. Safety of simultaneous bilateral total knee arthroplasty. A metaanalysis. J Bone Joint Surg Am. 2007; 89(6):1220-6.

14. Lombardi AV, Mallory TH, Fada RA, Hartman JF, Capps SG, Kefauver CA et al. Simultaneous bilateral total knee arthroplasties: who decides? Clin Orthop Relat Res. 2001; (392):319-29.

15. Rauh MA, Krackow KA. In-hospital deaths following elective total joint arthroplasty. Orthopaedics. 2004; 27(4):407-11.

16. Choi YJ, Lee HI, Ra HJ, Hwang DY, Kim TK, Shim SJ et al. Perioperative Risk Assessment in Patients Aged 75 Years or Older: Comparison between Bilateral and Unilateral Total Knee Arthroplasty. Knee Surg Relat Res. 2014; 26(4):222-9. 